\title{
Mechanism-Based Pharmacokinetic/Pharmacodynamic Modeling of the Glucagon-Like Peptide-1 Receptor Agonist Exenatide to Characterize Its Antiobesity Effects in Diet-Induced Obese Mice
}

\author{
Shinji Iwasaki, Teruki Hamada, Ikumi Chisaki, Tomohiro Andou, Noriyasu Sano, \\ Atsutoshi Furuta, and Nobuyuki Amano \\ Drug Metabolism and Pharmacokinetics Research Laboratories (S.I., T.H., I.C., N.S., A.F., N.A.) and Integrated Technology \\ Research Laboratories (T.A.), Takeda Pharmaceutical Co., Ltd., Kanagawa, Japan
}

Received May 4, 2017; accepted July 7, 2017

\begin{abstract}
In addition to their potent antidiabetic effects, glucagon-like peptide-1 (GLP-1) analogs lower body weight in humans. Hence, agonistic targeting of the GLP-1 receptor could be a valid approach to target obesity. However, quantitative analyses of the pharmacokinetic/pharmacodynamic (PK/PD) relationship between GLP-1 analogs and their antiobesity effect have not been reported in either animals or humans. Therefore, the present study was performed to establish a mechanism-based PK/PD model of GLP-1 receptor agonists using the GLP-1 analog exenatide for the development of promising new antiobesity drugs. Exenatide was administered to high-fat diet-induced obese C57BL/6J mice via subcutaneous bolus and continuous infusion. Food intake and body-weight reductions were observed and depended on the plasma concentrations of exenatide. The homeostatic feedback
\end{abstract}

model, in which food intake is assumed to be regulated by appetite control signals, described the relationship among the plasma concentration-time profile of exenatide, food intake, and body weight. The estimated $\mathrm{IC}_{50}$ of exenatide against food intake was $2.05 \mathrm{pM}$, which is similar to the reported $\mathrm{K}_{\mathrm{D}}$ value of exenatide in rat brain and the estimated $E_{50}$ value for augmentation of insulin secretion in humans. The PK/PD model simulation indicated that subcutaneous infusion would show a stronger effect on body-weight reduction than bolus dosing would. This novel, quantitative PK/PD model could be used for antiobesity research and development of GLP-1 analogs, GLP-1 secretagogues, GLP-1 degradation inhibitors, and combinations thereof by allowing the estimation of appropriate pharmacokinetic profiles and dosing regimens.

\section{Introduction}

Glucagon-like peptide-1 (GLP-1) is secreted from intestinal endocrine L-cells, which are located mainly in the distal ileum and colon. The active forms of GLP-1, GLP-1(7-37) and GLP$1(7-36) \mathrm{NH}_{2}$, have multiple biologic actions, including stimulation of insulin secretion in the pancreas, suppression of glucose production in the liver, delay of gastric emptying, and decrease of appetite (Baggio and Drucker, 2007). Owing to these biologic effects, GLP-1 is considered a promising target for the development of antidiabetes and antiobesity drugs. Hence, many GLP1 receptor agonists have been approved or introduced into clinical practice (Madsbad et al., 2011). Exenatide is one of the GLP-1 analogs, consisting of 39 amino acids and originally isolated from the saliva of the Gila monster. It has $53 \%$ sequence homology with human GLP-1 in its first 30 amino acids (Eng et al., 1992). Exenatide is more resistant to cleavage by dipeptidyl peptidase- 4 and has a longer half-life than native

The authors are full-time employees of Takeda Pharmaceutical Co., Ltd. https://doi.org/10.1124/jpet.117.242651.
GLP-1, owing to the difference in its amino acid sequence (Kolterman et al., 2005). Synthetic exenatide, referred to as exenatide, is approved as an antidiabetic drug because of its glucoregulatory functions, including glucose-dependent stimulation of insulin secretion, suppression of glucagon secretion, delay of gastric emptying, and protection of pancreatic $\beta$-cells (Young et al., 1999; Nielsen et al., 2004; Kolterman et al., 2005). In addition to the antidiabetic effects, exenatide decreased body weight by $0.7-2.5 \%$ at a dose of $10 \mu \mathrm{g}$ twice a day (BID) administered for 30 weeks (Buse et al., 2004; DeFronzo et al., 2005; Kendall et al., 2005). GLP-1 analogs reduce food intake via interaction with GLP-1 receptors localized in the hypothalamus, which regulates digestive behavior. In addition, GLP-1 analogs modify food intake indirectly by inhibiting gastric emptying, thereby producing gastric distension and an associated sensation of satiety (Baggio and Drucker, 2007). Therefore, the antiobesity effect of exenatide could be attributable to decreased food intake caused by appetite reduction.

A mechanism-based pharmacokinetic/pharmacodynamic (PK/PD) model can be used to quantitatively understand the relationship between drug concentrations and biologic responses

ABBREVIATIONS: AUC, area under the plasma concentration-time curve; DIO mice, high-fat diet-induced obese C57BL/6J mice; GLP-1, glucagon-like peptide-1; IIV, interindividual variability; LAR, long-acting release; LC-MS/MS, liquid chromatography-tandem mass spectrometry; PK/PD, pharmacokinetic/pharmacodynamics. 
(Mager et al., 2003). Hence, some PK/PD models of exenatide have been developed for evaluation of its antidiabetic effects. The effects of exenatide on glucose-insulin homeostasis were characterized under hyperglycemic clamping with a mechanism-based PD model and a hypothetical linear PK function (Mager et al., 2004). A target-mediated drug disposition (TMDD) PK/PD model of exenatide was developed to determine its effects on glucose-insulin homeostasis in type 2 diabetic Goto-Kakizaki rats (Gao and Jusko, 2011) and healthy rats (Gao and Jusko, 2012). Another TMDD PK/PD model of extended-release exenatide was established to characterize its effects on fasting plasma glucose and hemoglobin A1c in patients with type 2 diabetes mellitus (Li et al., 2015). These models contributed to the understanding of mechanism of action and the optimization of dosing regimens of exenatide.

Although PK/PD models of GLP-1 analogs for antidiabetic effect have been developed, no model to characterize its obesity effects has been reported to date. Hence, the present study was performed to establish a mechanism-based PK/PD model of exenatide to determine its antiobesity effects using high-fat diet-induced obese C57BL/6J mice (DIO mice). DIO mice have been widely used for the evaluation of the antiobesity effects of drugs, including exenatide (Arakawa et al., 2009), metformin (Matsui et al., 2010), dexfenfluramine, and sibutramine (Bush et al., 2006). We evaluated the plasma concentration-time profile of exenatide and the change in food intake over time after a single subcutaneous administration of exenatide to DIO mice. Additionally, the plasma concentrations of exenatide and the daily changes in food intake and body weight were evaluated in DIO mice during subcutaneous infusion of exenatide for 2 weeks. After the establishment of a pharmacokinetic model of exenatide, the relationship among the pharmacokinetics of exenatide, suppression of food intake, and reduction in body weight was modeled by fitting the data in the single-dose and 2-week infusion studies. The relationship between the pharmacokinetic profile and antiobesity effect was simulated by the established PK/PD model to estimate the appropriate pharmacokinetic profile of GLP-1 activity to maximize efficacy. In addition, species differences in the effect of exenatide on food intake between DIO mice and humans are discussed on the basis of our results and those published by others.

\section{Materials and Methods}

Materials and Reagents. Exenatide acetate was purchased from ChemPep Inc. (Wellington, FL). All other reagents and solvents were obtained from Wako Pure Chemicals Industries (Osaka, Japan).

Animals. The care and use of the animals and the experimental protocols used in this research were approved by the Institutional Animal Care and Use Committee of Shonan Research Center, Takeda Pharmaceutical Company Limited (Kanagawa, Japan, approval number 00005886). Male C57BL/6J mice (5 weeks old) were obtained from Charles River Laboratories Japan, Inc. (Kanagawa, Japan). DIO mice were established by feeding a high-fat diet (D12492; Research Diets Inc., New Brunswick, NJ) to the mice after 5 weeks of age. The experiments were conducted when the animals were 50-70 g (41-44 weeks old). Animals were kept housed in a temperature- and humidity-controlled room under 12-hour light/dark cycles.

Pharmacokinetic Studies. Exenatide dissolved in saline was administered subcutaneously to the DIO mice $(n=3)$ at doses of 10 and $100 \mathrm{nmol} / 2 \mathrm{ml}$ per kilogram. Blood samples were collected from the caudal vein $0.083,0.167,0.25,0.5,1,2,4$, and 8 hours after administration. Plasma samples were separated by centrifugation at $13,000 \mathrm{~g}$ and $4^{\circ} \mathrm{C}$ for 5 minutes. The concentrations of exenatide in plasma were analyzed by liquid chromatography-tandem mass spectrometry (LC-MS/MS).

Pharmacodynamic Studies. For evaluation of food intake after a single subcutaneous administration, exenatide dissolved in saline was subcutaneously injected into the DIO mice $(n=6-8)$ at doses of 0 (vehicle), 10 , and $100 \mathrm{nmol} / 2 \mathrm{ml}$ per kilogram just before the start of the dark phase (night time). The cumulative food intake was monitored every 2 hours for 38 hours. For evaluation of the food intake and body weights after the subcutaneous infusion for 2 weeks, exenatide dissolved in dimethyl sulfoxide/saline (1:1, v/v) was infused into the DIO mice ( $n=7$ ) at doses of 0 (vehicle), $0.3,1,3$, 10,30 , and $100 \mathrm{nmol} / \mathrm{kg}$ per day by a subcutaneously implanted Alzet micro-osmotic pump (model 1002; DURECT Corporation, Cupertino, CA). The Alzet pump was implanted in the DIO mice when the animals were 42 weeks old. Daily food intake and body weights were evaluated just before the start of the dark phase. Blood samples were collected from the caudal vein on day 7 of the infusion. Plasma samples were separated by centrifugation at $13,000 \mathrm{~g}$ and $4^{\circ} \mathrm{C}$ for 5 minutes. The concentrations of exenatide in plasma were analyzed by LC-MS/MS.

Quantification of Exenatide Concentration by LC-MS/MS. Aliquots $(50 \mu \mathrm{l})$ of the plasma samples diluted with control plasma were mixed with acetonitrile $(300 \mu \mathrm{l})$ containing the internal standard (IS). The mixtures were centrifuged at $4283 g$ and $4^{\circ} \mathrm{C}$ for 5 minutes. The supernatants were dried and then dissolved in acetonitrile/water/formic acid (50:49:1, v/v/v, $90 \mu \mathrm{l})$. The dissolved solutions $(5 \mu \mathrm{l})$ were injected into an LC-MS/MS system (API5000; AB Sciex, Framingham, MA). The chromatographic separations were performed on an XBridge BEH300 C18 column ( $3.5 \mu \mathrm{m}, 2.1 \times 50 \mathrm{~mm}$; Waters Corporation, Milford, MA) at $40^{\circ} \mathrm{C}$ with a gradient elution flow rate of $0.7 \mathrm{ml} / \mathrm{min}$. Mobile phase A (water/acetic acid, 100:0.2, v/v) and mobile phase B (acetonitrile/acetic acid, 100:0.2, v/v) were mixed for elution. Mobile phase B was held at 20\% for 0.5 minutes, and then increased linearly by $75 \%$ in 4 minutes. After mobile phase B was held at $95 \%$ for 2 minutes, it was returned to $20 \%$ in 0.01 minutes. Compounds were detected using multiple reaction monitoring in positive ionization mode [selected reaction monitoring (SRM) $\mathrm{m} / z=$ $838.3 \rightarrow 948.7]$. Analyst software TM (version 1.4.2; AB Sciex) was used for data acquisition and processing. The amount of exenatide in each plasma sample $(\mathrm{pg} / \mathrm{ml})$ was back-calculated using a calibration curve generated from a set of calibration standards. Linear calibration curves were constructed from plots of the peak area ratio of analyte to internal standard versus nominal analyte concentrations. The weighting factor $(1 / x)$ was determined on the basis of goodness-of-fit criteria, including the coefficient of determination, the back-calculated concentration of individual calibrators, and the minimizing intercept value.

Data Analysis. The modeling was performed using NONMEM VI (ICON Development Solutions, Ellicott City, MD) by means of the First-Order Conditional Estimation method with Interaction $(\mathrm{METHOD}=1, \mathrm{FOCE})$. The convergence criterion was three significant digits. A Compaq Digital Fortran Version 6.1 compiler was used to compile and execute NONMEM. Berkeley Madonna, ver. 8.3.8 (University of California, Berkeley, CA), was used for simulations. The interindividual variability (IIV) of the appropriate parameters was modeled using multiplicative exponential random effects as expressed in eq. 1 :

$$
P_{i}=\theta \cdot \exp \left(\eta_{i}\right)
$$

where $\theta$ is the typical population value for the parameter, $P_{i}$ is the individual prediction, and $\eta_{i}$ is the random deviation of $P_{i}$ from $\theta$. The values of $\eta_{\mathrm{i}}$ were assumed to be normally distributed, with a mean of zero and a variance of $\omega^{2}$. The derived IIV was expressed as the percent coefficient of variation CV. The precision of parameter estimates was expressed as the percentage of the ratio of the standard error divided by the parameter estimate (relative standard error, RSE). Residual variability was described using additive and proportional error models (eqs. 2 and 3, respectively): 


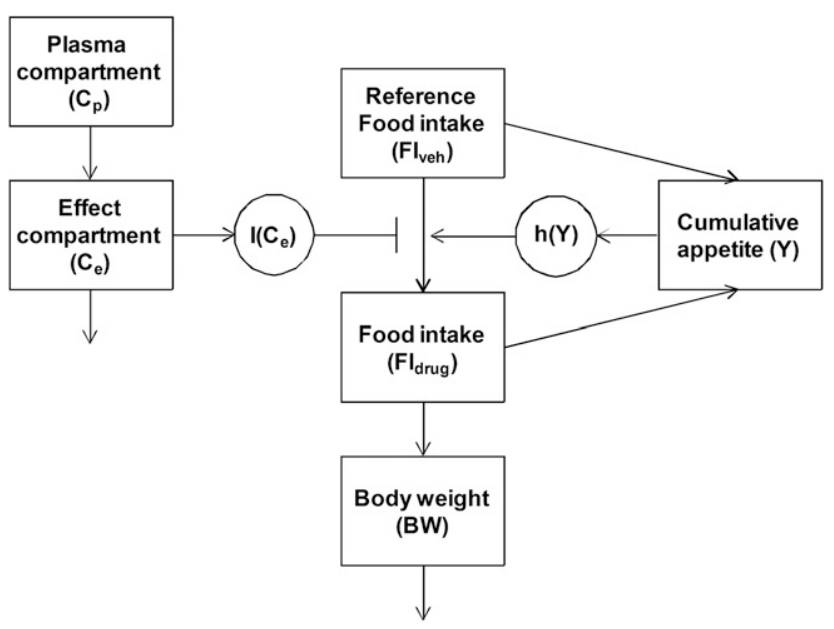

Fig. 1. Schematic of the models used to describe the PK/PD for food intake and body weight.

$$
\begin{aligned}
& Y_{i j}=F_{i j}+\varepsilon_{i j} \\
& Y_{i j}=F_{i j} \cdot\left(1+\varepsilon_{i j}\right)
\end{aligned}
$$

where $Y_{i j}$ denotes the observation for the $i^{\text {th }}$ individual at time $t_{j}, F_{i j}$ denotes the corresponding prediction as expressed by the model, and $\varepsilon_{\mathrm{ij}}$ denotes the residual random effect assumed to have a mean of zero and a variance of $\sigma^{2}$. Model selection was made on the basis of the visual inspection of goodness-of-fit plots, the precision of the parameter estimates, and the minimum value of the objective function.

Pharmacokinetic Modeling. The plasma concentration-time profile of exenatide was described by a one-compartment model with first-order absorption, as expressed in the following equations:

$$
\begin{gathered}
\frac{d X_{a}}{d t}=-k_{a} \cdot X_{a} \\
\frac{d C_{p}}{d t}=k_{a} \cdot X_{a} \cdot B A / V-k_{e l} \cdot C_{p}
\end{gathered}
$$

where $X_{a}$ is the drug amount in the absorption compartment, $C_{p}$ is the plasma concentration of exenatide, $k_{a}$ is the absorption rate constant, $B A / V$ is the bioavailability divided by the distribution volume of the plasma concentration compartment, and $k_{e l}$ is the elimination rate constant from the plasma concentration compartment.

Pharmacodynamic Modeling. The general scheme of the applied PK/PD model is presented in Fig. 1.

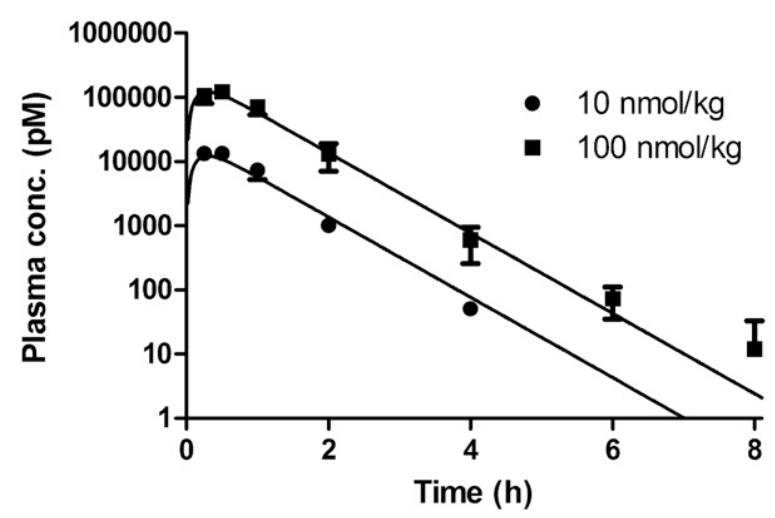

Fig. 2. Plasma concentration-time profiles of exenatide after subcutaneous administration of 10 and $100 \mathrm{nmol} / \mathrm{kg}$ in DIO mice. Symbols represent mean observed values (mean \pm S.D., $n=3$ ), and solid lines indicate the population predicted profiles.
The food intake of the vehicle group $\left(F I_{v e h}\right)$ was high during the dark phase and low during the light phase. The $F I_{v e h}$ in the single dosing study was described by monoexponential decay with the following equation:

$$
F I_{v e h}=p 1 \cdot \exp (-p 2 \cdot t)
$$

where $p 1$ is the initial level of food intake and $p 2$ is the constant to represent the monoexponential decline in food intake. Since it was technically difficult to monitor the food intake every few hours for 2 weeks in the infusion study, we assumed that $F I_{v e h}$ in the infusion study was a constant value $\left(F I_{\text {veh,inf }}\right)$.

To describe the longer duration of food intake suppression compared with the elimination of the plasma concentration of exenatide in the single-dosing study, the effect compartment was introduced by the following equation:

$$
\frac{d C_{e}}{d t}=k_{e o} \cdot C_{p}-k_{e o} \cdot C_{e}
$$

where $C_{e}$ is the concentration of exenatide in the hypothetical effect compartment and $k_{e o}$ is the elimination rate constant from the effect compartment.

In reference to the report by Gennemark et al. (2015), a homeostatic feedback model was applied for the food-intake modeling. The food intake of the dosed group $\left(F I_{d r u g}\right)$ was assumed to be determined by the inhibitory effect of exenatide on the food intake, $I\left(C_{e}\right)$, and the appetite control signal $(h(Y))$ as described in the following equations:

$$
\begin{aligned}
& F I_{\text {drug }}=F I_{\text {veh }} \cdot I\left(C_{e}\right) \cdot h(Y) \\
& I\left(C_{e}\right)=1-\frac{I_{\max } \cdot C_{e}{ }^{H i l l}}{I C_{50}{ }^{H i l l}+C_{e}{ }^{H i l l}} \\
& h(Y)=h_{\min }+\left(h_{\max }-h_{\min }\right) \cdot\left(\frac{1-h_{\min }}{h_{\max }-h_{\min }}\right)^{\exp \left(-h_{\text {slope }} \cdot Y\right)} \\
& \frac{d Y}{d t}=\left(F I_{v e h}-F I_{d r u g}\right)
\end{aligned}
$$

where $I_{\max }$ is the maximum inhibition potential of exenatide for the food intake suppression, $\mathrm{IC}_{50}$ is the concentration in effect compartment that produces $50 \%$ of $I_{\max }$, Hill is the Hill exponent, $h_{\min }$ is the lower asymptote of $h(Y), h_{\max }$ is the upper asymptote of $h(Y)$, and $h_{\text {slope }}$ is the slope of $h(Y)$. The function $h(Y)$ is set to 1 when there is no imbalance in food intake and $Y=0$. The $h_{\max }$ is related to the increase of appetite followed by the food-intake suppression. The $h_{\min }$ is related to the decrease in appetite by overfeeding.

The body weight of mice $(B W)$ was assumed to be controlled by energy expenditure, which was determined by the basal body weight $\left(B W_{\text {base }}\right)$ and the amount of food intake as described in the following equation:

$$
B W=B W_{b a s e}-p 3 \cdot B W_{b a s e}+p 4 \cdot \int_{o}^{t} F I_{d r u g}
$$

where $p 3$ is the constant that produces the body-weight reduction by energy expenditure and $p 4$ is the conversion efficiency of food intake into body weight.

TABLE 1

Estimated pharmacokinetic parameters of exenatide in DIO mice after a single subcutaneous injection

Relative standard errors (\%) are shown in parentheses after the estimated values.

\begin{tabular}{llc}
\hline \multicolumn{1}{c}{ Parameter } & \multicolumn{1}{c}{ Definition } & Estimate \\
\hline$k_{a}\left(\mathrm{~h}^{-1}\right)$ & Absorption rate & $6.39(6.59)$ \\
$k_{e l}\left(\mathrm{~h}^{-1}\right)$ & Elimination rate & $1.44(5.99)$ \\
$B A / V(\mathrm{~kg} / \mathrm{l})$ & Bioavailability divided by & $1.89(9.68)$ \\
& distribution volume & \\
IIV of $k_{e l}(\%)$ & & $11.6(59.2)$ \\
Residual error $(\%)$ & & $32.2(19.3)$ \\
\hline
\end{tabular}


TABLE 2

Plasma concentration (pM) of exenatide in DIO mice on day 7 during subcutaneous infusion $(n=7)$

\begin{tabular}{rrr}
\hline \multicolumn{1}{c}{ Dose } & \multicolumn{1}{c}{ Mean } & \multicolumn{1}{c}{ S.D. } \\
\hline nmol/kg per day & & \\
0.3 & 9.1 & 7.3 \\
1 & 39.8 & 24.7 \\
3 & 116.3 & 33.6 \\
10 & 521.6 & 182.1 \\
30 & 747.7 & 383.6 \\
100 & 8236.5 & 3549.3 \\
\hline
\end{tabular}

\section{Results}

Pharmacokinetics of Exenatide in DIO Mice. The plasma concentration-time profiles of exenatide after subcutaneous dosing of 10 and $100 \mathrm{nmol} / \mathrm{kg}$ in DIO mice were modeled (Fig. 2 and Table 1). Exenatide was rapidly absorbed following subcutaneous dosing and reached a maximum concentration within 0.5 hours. By simultaneously fitting all the data, the time profiles of plasma concentration were well described by a one-compartment model with first-order absorption and linear elimination. The RSE values for estimated parameters were between 5.99 and $9.68 \%$. The IIV of $k_{e l}$ was $11.6 \%$, and residual error was $32.2 \%$.

The plasma concentrations of exenatide on day 7 during subcutaneous infusion at doses of $0.3,1,3,10,30$, and $100 \mathrm{nmol} / \mathrm{kg}$ per day in DIO mice are shown in Table 2 . The plasma concentrations increased almost in a dose-dependent manner, except for those at a dose of $30 \mathrm{nmol} / \mathrm{kg}$ per day. Since the measured individual plasma concentrations of exenatide were used for PK/PD modeling in the subcutaneous infusion study, the plasma concentrations during subcutaneous infusion were not included in the PK modeling.

Pharmacodynamics of Exenatide in DIO Mice. The time profiles of cumulative food intake after a single subcutaneous administration of vehicle or exenatide at doses of 10 and $100 \mathrm{nmol} / \mathrm{kg}$ are shown in Fig. 3. Food intake was suppressed in a dose-dependent manner immediately after the administration of exenatide. The suppression ratios of cumulative food intake at 24 hours after the administration of 10 and $100 \mathrm{nmol} / \mathrm{kg}$ exenatide (against that of vehicle treatment group) were 58.8 and $76.5 \%$, respectively.

The changes in daily food intake and body weight during subcutaneous infusion of vehicle or exenatide at doses of $0.3,1$, $3,10,30$, and $100 \mathrm{nmol} / \mathrm{kg}$ per day are shown in Figs. 4 and 5, respectively. The daily food intake was suppressed in a dosedependent manner. The effect of food intake suppression was strongest on day 1 and then gradually decreased with time at all doses. The suppression ratios of cumulative food intake on day 14 at doses of $0.3,1,3,10,30$, and $100 \mathrm{nmol} / \mathrm{kg}$ per day against that of vehicle treatment group were 4.9, 10.6, 22.8, $32.7,31.0$, and $36.5 \%$, respectively. The body weights of the DIO mice infused with vehicle were stable during the experiment but decreased during infusion of exenatide, depending on the degree of food intake suppression. The ratio of body-weight reduction on day 14 after infusion of exenatide at doses of $0.3,1,3,10,30$, and $100 \mathrm{nmol} / \mathrm{kg}$ per day against that of vehicle treatment was $1.9,2.7,9.2,13.1,12.5$, and $15.7 \%$, respectively.

As shown in Figs. 3 and 4, the homeostatic feedback model combined with the effect compartment adequately characterized the food intake changes by exenatide in DIO mice both after single subcutaneous administration and during subcutaneous infusion. In addition, the body-weight changes in the infusion study were adequately characterized by the model described in eq. 12, as shown in Fig. 5. The estimated pharmacodynamic parameters are summarized in Table 3. The introduction of the effect compartment was crucial to characterization of food intake changes after single subcutaneous administration, since the duration of food intake suppression was longer than that of the plasma concentrations of exenatide. The elimination rate constant from the effect compartment $\left(k_{e o}\right)$ was estimated to be 0.344 hour $^{-1}$, which was one-fourth of the $k_{e l}$. The $I_{\max }$ was assumed to be 1 since full suppression of food intake was observed in the early phase after single subcutaneous administration of exenatide. The $\mathrm{IC}_{50}$ value for food intake suppression was estimated to be $2.05 \mathrm{pM}$.
A

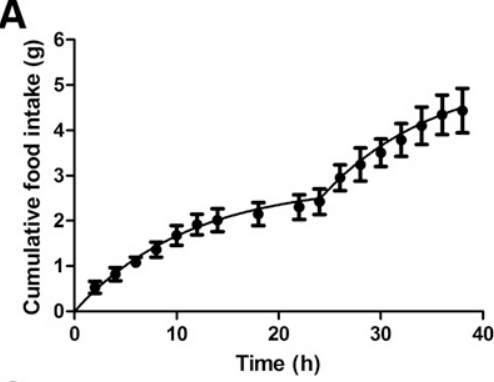

C

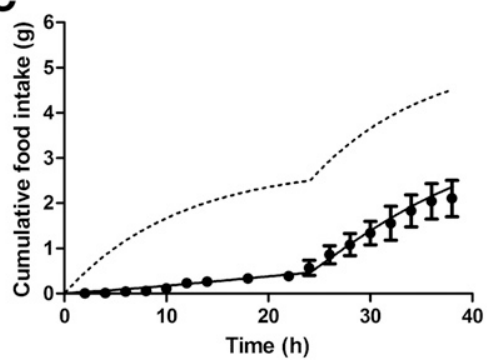

\section{B}

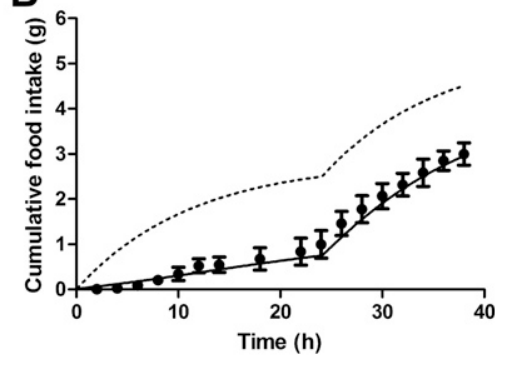

Fig. 3. Time profiles of cumulative food intake after a single subcutaneous administration of vehicle (A) or exenatide at doses of 10 (B) and $100 \mathrm{nmol} / \mathrm{kg}$ (C) in DIO mice. Symbols represent mean observed values (mean \pm S.D., $n=6-8$ ), solid lines indicate the population predicted profiles, and the dashed lines represent the population predicted profiles of the vehicle group. 
A

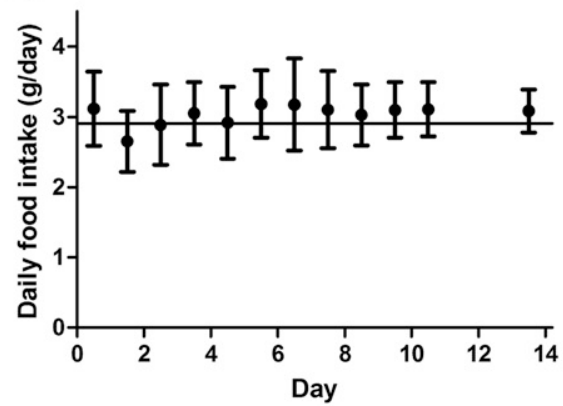

C

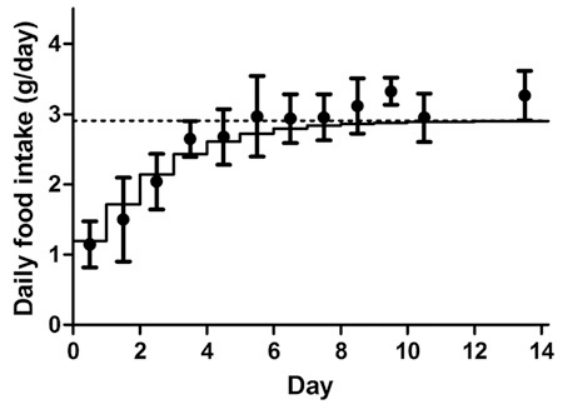

E

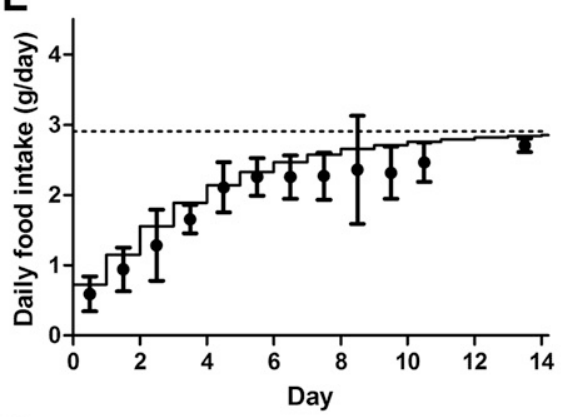

G

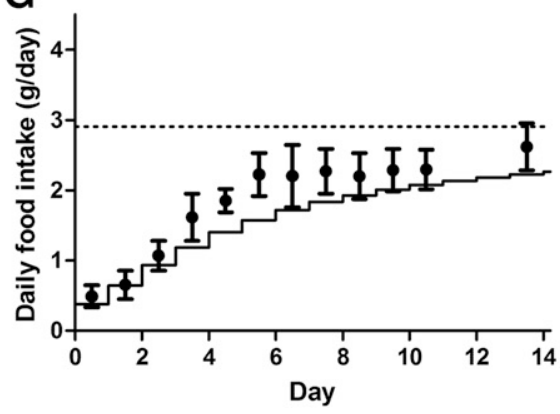

B

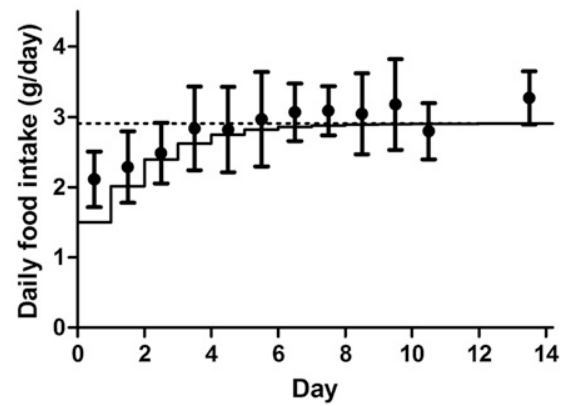

D

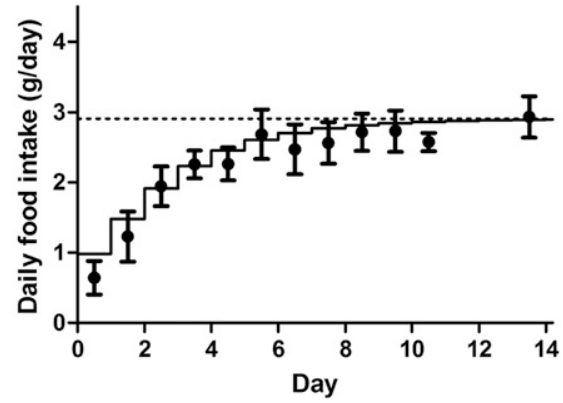

$\mathbf{F}$

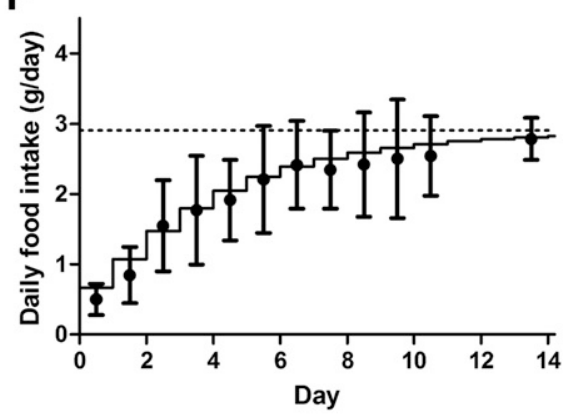

Fig. 4. Changes in daily food intake during subcutaneous infusion of vehicle (A) or exenatide at doses of $0.3(\mathrm{~B}), 1$ (C), 3 (D), 10 (E), 30 (F), and $100 \mathrm{nmol} / \mathrm{kg}$ per day (G) in DIO mice. Symbols represent mean observed values (mean \pm S.D., $n=7$ ), solid lines indicate the population predicted profiles, and the dashed lines represent the population predicted profiles of the vehicle group.
Simulation of the Relationship between the Pharmacokinetics and Pharmacodynamics. To discuss the ideal pharmacokinetic profile of exenatide for antiobesity effects, the relationship between the daily area under the plasma concentration-time curve (AUC) of exenatide for plasma and body-weight reduction after multiple subcutaneous administrations and subcutaneous infusion for 2 weeks was simulated by the PK/PD model. To mimic human biorhythms, the time profile of daily food intake was assumed to be three times a day, at 0,6 , and 12 hours, with a duration of 0.5 hours per feeding period. In the multiple subcutaneous administration simulation, exenatide was dosed BID every 12 hours, just before the first and third meal. As shown in Fig. 6, subcutaneous infusion required a lower exenatide plasma exposure for any amount of body-weight reduction compared with that of subcutaneous bolus administration. For example, the required AUC for a 15\% reduction in body weight by multiple bolus administrations and continuous infusion was 1181 and $621 \mathrm{nmol} \cdot \mathrm{h} / \mathrm{l}$, respectively.

\section{Discussion}

GLP-1 analogs, including exenatide, show antiobesity effects in animals (Arakawa et al., 2009) and humans (Madsbad, 2009); however, their PK/PD models have not been reported. This is the first study to establish a mechanism-based PK/PD 


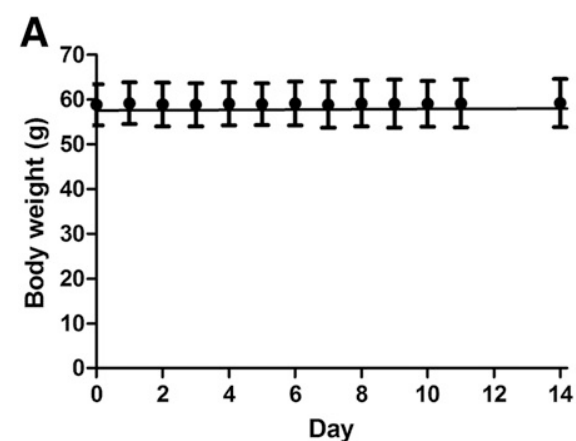

C
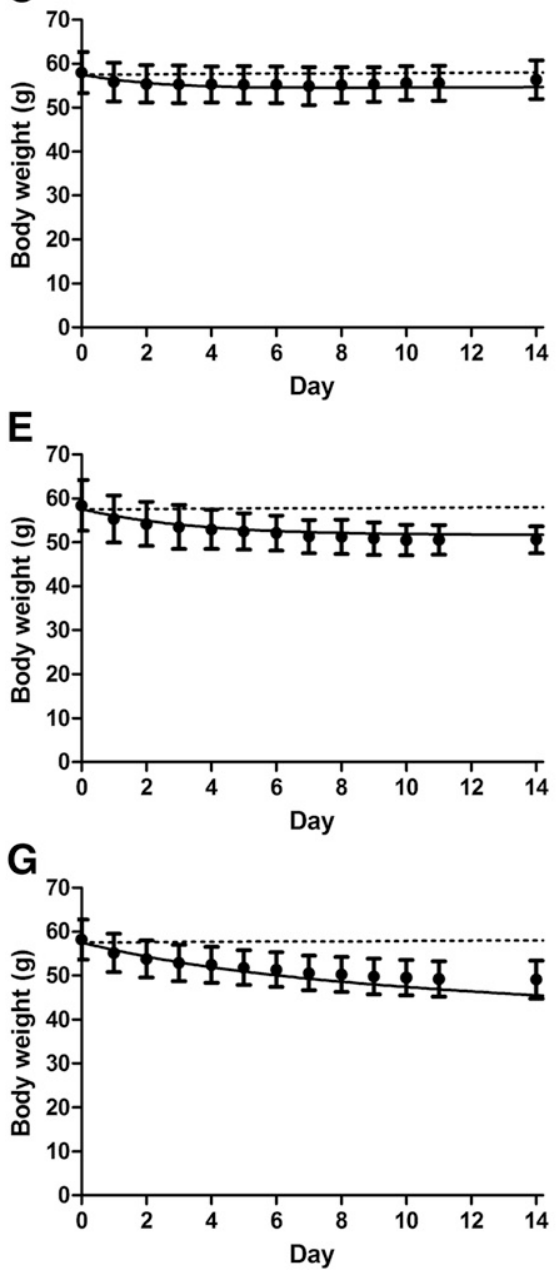

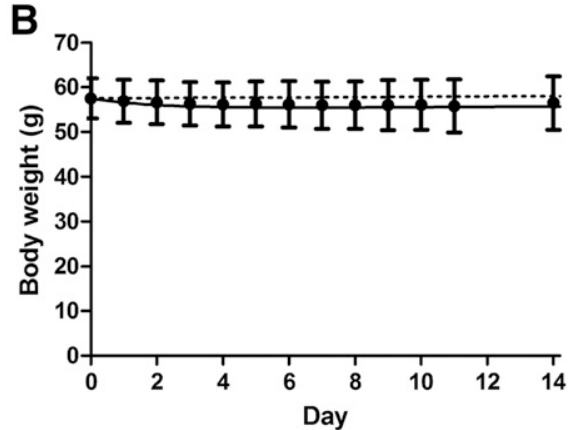

D
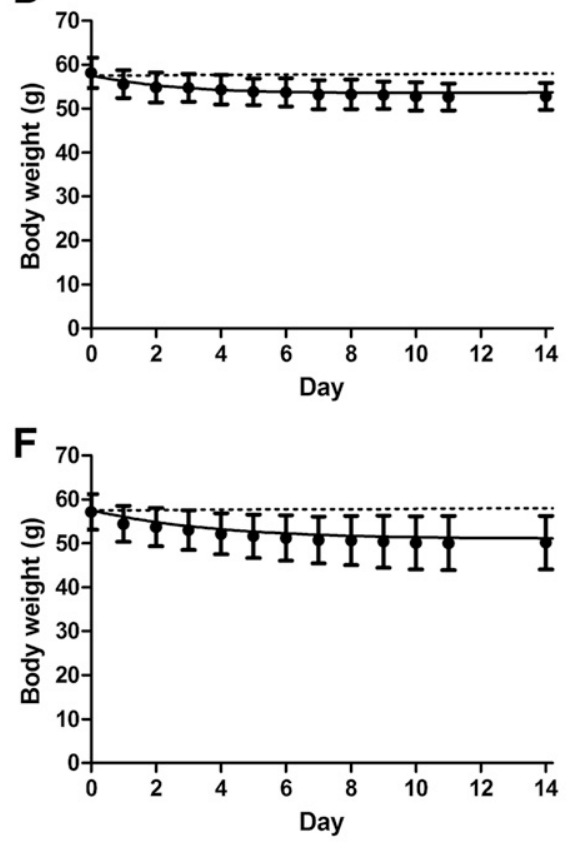

Fig. 5. Changes in body weight during subcutaneous infusion of vehicle (A) or exenatide at doses of $0.3(\mathrm{~B}), 1(\mathrm{C}), 3(\mathrm{D}), 10(\mathrm{E}), 30(\mathrm{~F})$, and $100 \mathrm{nmol} / \mathrm{kg}$ per day (G) in DIO mice. Symbols represent mean observed values (mean \pm S.D., $n=7$ ), solid lines indicate the population predicted profiles, and the dashed lines represent the population predicted profiles of the vehicle group. model of a GLP-1 analog to determine its antiobesity effect in animals. In patients with type 2 diabetes, the administration of the long-acting release formulation of exenatide (exenatide LAR) at a dose of $2 \mathrm{mg} /$ week for 15 weeks produced a bodyweight reduction of $3.4 \%$ (Kim et al., 2007). In this report, the plasma concentration of exenatide was at a steady state from week 6 , and the average concentration of exenatide for 15 weeks was approximately $40 \mathrm{pM}$. Another study in overweight individuals showed an approximately $8 \%$ body-weight reduction with a $25 \%$ calorie restriction for 15 weeks (Heilbronn et al., 2006). Assuming that the calorie restriction and body-weight reduction correlate proportionally, the $3.4 \%$ body-weight reduction by exenatide LAR at $2 \mathrm{mg} /$ week for 15 weeks would be attributable to the decrease in caloric intake by $10.7 \%$. These observations suggest that a plasma concentration of $40 \mathrm{pM}$ in humans would result in food intake suppression by $10.7 \%$. In our study, the subcutaneous infusion of exenatide at a dose of $1 \mathrm{nmol} / \mathrm{kg}$ per day for 2 weeks in DIO mice suppressed food intake by $9.8 \%$ with a plasma concentration of exenatide at $39.8 \mathrm{pM}$. In humans and DIO mice, $40 \mathrm{pM}$ exenatide in plasma results in food intake suppression by approximately $10 \%$ for 15 weeks and 2 weeks, respectively. These results suggested that the effect of exenatide on food intake suppression in DIO mice for 2 weeks could be used as a surrogate of long-term (15-week) human response. However, adequate preclinical-to-clinical translation of food intake suppression effect remains challenging owing to the complexity of food intake regulation. Food intake is regulated by a 
TABLE 3

Estimated pharmacodynamic parameters of exenatide for food-intake suppression and body-weight reduction in DIO mice

Relative standard errors (\%) are shown in parentheses after the estimated values.

\begin{tabular}{|c|c|c|}
\hline Parameter & Estimate & Definition \\
\hline$p 1$ (g) & $0.251(6.57)$ & Initial level of food intake in single administration study \\
\hline$p 2\left(\mathrm{~h}^{-1}\right)$ & $0.0884(10.7)$ & $\begin{array}{l}\text { Constant to represent the monoexponential decline of food } \\
\text { intake in single-administration study }\end{array}$ \\
\hline$B W_{\text {base }}(\mathrm{g})$ & $57.5(1.1)$ & Basal body weight of mice \\
\hline$p 3$ & $0.00140(2.09)$ & Constant that produces the body-weight reduction \\
\hline$p 4$ & $0.677(2.79)$ & Conversion efficiency of food intake into body weight \\
\hline$k_{e o}\left(\mathrm{~h}^{-1}\right)$ & $0.344(11.2)$ & Elimination rate constant from effect compartment \\
\hline Hill & $0.291(14.8)$ & Hill exponent/sigmoidicity factor \\
\hline$h_{\min }$ & 0 Fix $^{a}$ & Lower asymptote of $h(Y)$ \\
\hline$h_{\max }$ & $11.2(9.91)$ & Upper asymptote of $h(Y)$ \\
\hline$h_{\text {slope }}$ & $0.143(12.5)$ & Slope of $h(Y)$ \\
\hline IIV of $p 1(\%)$ & $9.5(46.7)$ & \\
\hline IIV of $F I_{\text {veh }}(\%)$ & $12.3(25.0)$ & \\
\hline IIV of $B L_{\text {base }}(\%)$ & $7.3(19.7)$ & \\
\hline $\begin{array}{l}\text { Residual error (\%) for body weight during } \\
\text { subcutaneous infusion }\end{array}$ & $0.7(8.4)$ & \\
\hline
\end{tabular}

${ }^{a}$ Fix, parameter fixed at the stated value.

number of factors including energetic state of the organism, gastrointestinal hormonal and neuronal signals, nutrients, time of day, food availability, and palatability. In addition to these factors, disease state and genetic and ethnic differences would also cause interindividual difference in efficacy in humans. Building a mechanistic model incorporating all those factors should be the next step to facilitate the preclinical-to-clinical translation of food regulation. The model established in this study would represent a basic structure that could serve as a future foundation of more mechanistic translational models for antiobesity.

The $\mathrm{IC}_{50}$ value of exenatide against food intake suppression in DIO mice was estimated to be $2.05 \mathrm{pM}$ in our study (Table 3). It has been reported that exenatide has high- and low-affinity binding sites in the posterior pituitary lobe in rats (Goke et al., 1995). The $\mathrm{K}_{\mathrm{D}}$ values for these sites were 6.2 and $930 \mathrm{pM}$, respectively. The lower $\mathrm{K}_{\mathrm{D}}$ value in the rat pituitary lobe was close to the $\mathrm{IC}_{50}$ value in DIO mice that we found. A mechanism-based PK/PD model was developed to characterize the effect of exenatide on glucose-insulin homeostasis in healthy and type 2 diabetic subjects (Mager et al., 2004). The reported $\mathrm{EA}_{50}$ values, the amount of exenatide producing $50 \%$ of $\mathrm{E}_{\max }$, for the augmentation of insulin secretion in healthy and type 2 diabetic subjects were 0.268 and $0.111 \mathrm{pmol} / \mathrm{kg}$, respectively. By dividing the $\mathrm{EA}_{50}$ values by the distribution volume $(\mathrm{Vd})$ of exenatide, which was reported to be $64 \mathrm{ml} / \mathrm{kg}$ (Edwards et al., 2001), the $\mathrm{EC}_{50}$ values of exenatide for the augmentation of insulin secretion in healthy and type 2 diabetic subjects could be estimated at 4.2 and $1.7 \mathrm{pM}$, respectively. The estimated $\mathrm{EC}_{50}$ value for the augmentation of insulin secretion in humans was similar to the $\mathrm{IC}_{50}$ value in our DIO mouse study. These results suggest that the $\mathrm{EC}_{50}$ of exenatide in humans for the antiobesity effect would be similar to that for the antidiabetic effect.

The suppressive effect of exenatide on food intake in the subcutaneous infusion study gradually decreased at all doses
(Fig. 4). To clarify whether the decrease of $\mathrm{E}_{\max }$ was the reason for the attenuation, a large dose of exenatide (1000 $\mathrm{nmol} / \mathrm{kg})$ was administered subcutaneously to DIO mice immediately after the 2-week subcutaneous infusion. As a result, food intake was almost completely suppressed in all groups, except the highest dose group (data not shown). This observation indicates that the decrease in $\mathrm{E}_{\max }$ was not the cause of the attenuation of food intake suppression. Therefore, we adopted a homeostatic feedback model described in eqs. 8-11 on the basis of work by Gennemark et al. (2015). The model assumed that the body increased appetite in response to the food intake suppression by exenatide to maintain the energy intake level to live. The baseline of appetite was controlled by the total amount of food intake. The cumulative imbalance in food intake between vehicle and drug-treated groups, which was defined as $Y$, was calculated by eq. 11. The imbalance in food intake was then transformed into the appetite control signal

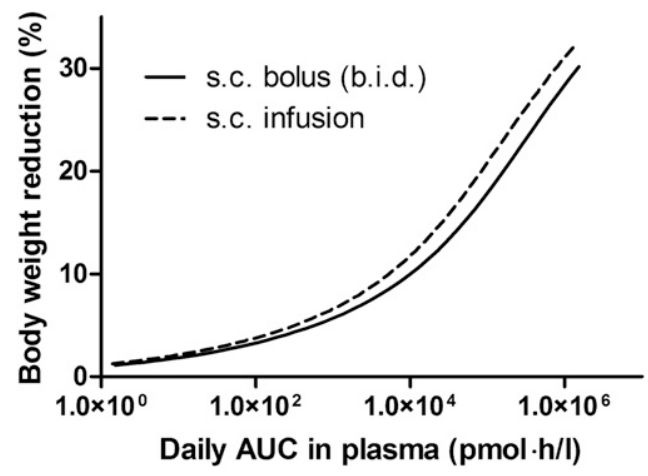

Fig. 6. Simulated relationship between daily AUC in plasma of exenatide and body-weight reduction after multiple subcutaneous administrations (BID) and subcutaneous infusion for 2 weeks in DIO mice. 
denoted by $h(Y)$ (eq. 10). The appetite control signal competed with the food intake suppression effect by exenatide treatment, which resulted in the attenuation of the efficacy with time (eq. 8). Since there was no overfeeding in our study, it was technically difficult to estimate the $h_{\text {min }}$, which is the lower limit of appetite feedback. Hence, $h_{\min }$ was assumed to be 0 . The homeostatic feedback model well described the time profile of food intake in both the single-dosing and infusion studies.

The relationship between the daily AUC of exenatide in plasma and the effect on body-weight reduction in DIO mice was compared between subcutaneous BID administration and infusion, by simulation in the established PK/PD model (Fig. 6 ). The simulation indicated that subcutaneous infusion would have a stronger effect on body-weight reduction than bolus dosing did at the same level of daily AUC of exenatide. For example, the body-weight reduction by infusion, which achieves $10,000 \mathrm{pmol}^{*} \mathrm{~h} / \mathrm{l}$ daily AUC of exenatide, was simulated to be $10.2 \%$ and that by bolus dosing was simulated to be $8.7 \%$. These results suggest that GLP-1 agonists with a long half-life in the plasma concentration-time profile would be more efficacious than those with a short half-life for achieving a body-weight reduction target. Currently, two types of formulation of exenatide, exenatide and exenatide LAR, are available for clinical use. The plasma concentration of exenatide after subcutaneous dosing of exenatide at $0.1 \mu \mathrm{g} / \mathrm{kg}$ reached its $\mathrm{C}_{\max }$ at 2 hours and disappeared within 15 hours (Kolterman et al., 2005). On the other hand, the plasma concentration of exenatide LAR at $2 \mathrm{mg} /$ week was at a steady state from the 6th week onward (Kim et al., 2007). These formulations with different pharmacokinetic profiles are useful for understanding the pharmacokinetic profile of the antiobesity effect of exenatide in humans. Many clinical studies of exenatide (Buse et al., 2004; DeFronzo et al., 2005; Heine et al., 2005; Kendall et al., 2005; Nauck et al., 2007; Zinman et al., 2007; Apovian et al., 2010) and exenatide LAR (Kim et al., 2007; Bergenstal et al., 2010; Fineman et al., 2011) have been reported. However, it is difficult to compare the $\mathrm{PK} / \mathrm{PD}$ across studies quantitatively since each study had a different population of subjects with different treatments. Drucker et al. (2008) directly compared the efficacy of exenatide LAR ( $2 \mathrm{mg} /$ week) to that of exenatide administered twice a day (10 $\mu \mathrm{g}$, BID) for 30 weeks in subjects with type 2 diabetes. However, the degrees of body-weight reduction by exenatide LAR and exenatide in that study were not significantly different from the placebo group (3.6 and 3.7\%, respectively, $p>0.05$ ). Hence, these results would not be suitable for the quantitative PK/PD analysis. Although our model was established on the basis of data that were not from humans, it would be useful to estimate the appropriate dosing regimen and formulation for the antiobesity effect not only of exenatide but also of other GLP-1 analogs.

In conclusion, we developed a mechanism-based PK/PD model of exenatide to determine its antiobesity effects in DIO mice. Whereas the developed model may not be able to predict the pharmacological effects of other antiobesity drugs with mechanisms of action different from that of exenatide, the model could be applied to estimate the appropriate pharmacokinetic profiles and dosing regimens of GLP-1 analogs for antiobesity treatment. In addition, the model could be used for research and development of GLP-1 secretagogues, dipeptidyl peptidase- 4 inhibitors, and evaluation of their combined antiobesity effects by incorporating the differences in pharmacokinetics and GLP-1 receptor agonistic activity of exenatide and GLP-1 into the model.

\section{Acknowledgments}

The authors thank Dr. Joost de Jongh (Leiden Experts on Advanced Pharmacokinetics and Pharmacodynamics) and Akihiko Goto for technical assistance and many thoughtful suggestions.

\section{Authorship Contributions}

Participated in research design: Iwasaki, Hamada, Chisaki, Amano.

Conducted experiments: Iwasaki, Hamada, Chisaki, Andou, Sano, Furuta.

Performed data analysis: Iwasaki, Hamada, Chisaki.

Wrote or contributed to the writing of the manuscript: Iwasaki, Hamada, Chisaki, Andou, Sano, Furuta, Amano.

\section{References}

Apovian CM, Bergenstal RM, Cuddihy RM, Qu Y, Lenox S, Lewis MS, and Glass LC (2010) Effects of exenatide combined with lifestyle modification in patients with type 2 diabetes. Am J Med 123:468 e9-e17.

Arakawa M, Ebato C, Mita T, Hirose T, Kawamori R, Fujitani Y, and Watada H (2009) Effects of exendin-4 on glucose tolerance, insulin secretion, and beta-cell proliferation depend on treatment dose, treatment duration and meal contents. Biochem Biophys Res Commun 390:809-814.

Baggio LL and Drucker DJ (2007) Biology of incretins: GLP-1 and GIP. Gastroenterology 132:2131-2157.

Bergenstal RM, Wysham C, Macconell L, Malloy J, Walsh B, Yan P, Wilhelm K, Malone J, and Porter LE; DURATION-2 Study Group (2010) Efficacy and safety of exenatide once weekly versus sitagliptin or pioglitazone as an adjunct to metformin for treatment of type 2 diabetes (DURATION-2): a randomised trial. Lancet 376: 431-439.

Buse JB, Henry RR, Han J, Kim DD, Fineman MS, and Baron AD; Exenatide-113 Clinical Study Group (2004) Effects of exenatide (exendin-4) on glycemic control over 30 weeks in sulfonylurea-treated patients with type 2 diabetes. Diabetes Care 27:2628-2635.

Bush EN, Shapiro R, Brune ME, Knourek-Segel VE, Droz BA, Fey T, Lin E, and Jacobson PB (2006) Chronic treatment with either dexfenfluramine or sibutramine in diet-switched diet-induced obese mice. Endocrine 29:375-381.

DeFronzo RA, Ratner RE, Han J, Kim DD, Fineman MS, and Baron AD (2005) Effects of exenatide (exendin-4) on glycemic control and weight over 30 weeks in metformin-treated patients with type 2 diabetes. Diabetes Care 28:1092-1100.

Drucker DJ, Buse JB, Taylor K, Kendall DM, Trautmann M, Zhuang D, and Porter L; DURATION-1 Study Group (2008) Exenatide once weekly versus twice daily for the treatment of type 2 diabetes: a randomised, open-label, non-inferiority study. Lancet 372:1240-1250.

Edwards CM, Stanley SA, Davis R, Brynes AE, Frost GS, Seal LJ, Ghatei MA, and Bloom SR (2001) Exendin-4 reduces fasting and postprandial glucose and decreases energy intake in healthy volunteers. Am J Physiol Endocrinol Metab 281:E155-E161.

Eng J, Kleinman WA, Singh L, Singh G, and Raufman JP (1992) Isolation and characterization of exendin-4, an exendin-3 analogue, from Heloderma suspectum venom. Further evidence for an exendin receptor on dispersed acini from guinea pig pancreas. J Biol Chem 267:7402-7405.

Fineman M, Flanagan S, Taylor K, Aisporna M, Shen LZ, Mace KF, Walsh B, Diamant M, Cirincione B, Kothare P, et al. (2011) Pharmacokinetics and pharmacodynamics of exenatide extended-release after single and multiple dosing. Clin Pharmacokinet 50:65-74

Gao W and Jusko WJ (2011) Pharmacokinetic and pharmacodynamic modeling of exendin- 4 in type 2 diabetic Goto-Kakizaki rats. $J$ Pharmacol Exp Ther 336: $881-890$.

Gao W and Jusko WJ (2012) Target-mediated pharmacokinetic and pharmacodynamic model of exendin-4 in rats, monkeys, and humans. Drug Metab Dispos 40: 990-997.

Gennemark P, Hjorth S, and Gabrielsson J (2015) Modeling energy intake by adding homeostatic feedback and drug intervention. J Pharmacokinet Pharmacodyn 42 : 79-96.

Göke R, Larsen PJ, Mikkelsen JD, and Sheikh SP (1995) Identification of specific binding sites for glucagon-like peptide-1 on the posterior lobe of the rat pituitary. Neuroendocrinology 62:130-134.

Heilbronn LK, de Jonge L, Frisard MI, DeLany JP, Larson-Meyer DE, Rood J, Nguyen T, Martin CK, Volaufova J, Most MM, et al.; Pennington CALERIE Team (2006) Effect of 6-month calorie restriction on biomarkers of longevity, metabolic adaptation, and oxidative stress in overweight individuals: a randomized controlled trial. JAMA 295:1539-1548.

Heine RJ, Van Gaal LF, Johns D, Mihm MJ, Widel MH, and Brodows RG; GWAA Study Group (2005) Exenatide versus insulin glargine in patients with suboptimally controlled type 2 diabetes: a randomized trial. Ann Intern Med 143: 559-569.

Kendall DM, Riddle MC, Rosenstock J, Zhuang D, Kim DD, Fineman MS, and Baron $\mathrm{AD}$ (2005) Effects of exenatide (exendin-4) on glycemic control over 30 weeks in patients with type 2 diabetes treated with metformin and a sulfonylurea. Diabetes Care 28:1083-1091. 
Kim D, MacConell L, Zhuang D, Kothare PA, Trautmann M, Fineman M, and Taylor K (2007) Effects of once-weekly dosing of a long-acting release formulation of exenatide on glucose control and body weight in subjects with type 2 diabetes. Diabetes Care $\mathbf{3 0}$ 1487-1493.

Kolterman OG, Kim DD, Shen L, Ruggles JA, Nielsen LL, Fineman MS, and Baron $\mathrm{AD}$ (2005) Pharmacokinetics, pharmacodynamics, and safety of exenatide in patients with type 2 diabetes mellitus. Am J Health Syst Pharm 62:173-181.

$\mathrm{Li} \mathrm{H}, \mathrm{Xu}$ J, and Fan X (2015) Target-mediated pharmacokinetic/pharmacodynamic model based meta-analysis and dosing regimen optimization of a long-acting release formulation of exenatide in patients with type 2 diabetes mellitus. J Pharmacol Sci 127:170-180.

Madsbad S (2009) Exenatide and liraglutide: different approaches to develop GLP-1 receptor agonists (incretin mimetics)-preclinical and clinical results. Best Pract Res Clin Endocrinol Metab 23:463-477.

Madsbad S, Kielgast U, Asmar M, Deacon CF, Torekov SS, and Holst JJ (2011) An overview of once-weekly glucagon-like peptide-1 receptor agonists-available efficacy and safety data and perspectives for the future. Diabetes Obes Metab 13:394-407.

Mager DE, Abernethy DR, Egan JM, and Elahi D (2004) Exendin-4 pharmacodynamics: insights from the hyperglycemic clamp technique. J Pharmacol Exp Ther 311:830-835. Mager DE, Wyska E, and Jusko WJ (2003) Diversity of mechanism-based pharmacodynamic models. Drug Metab Dispos 31:510-518.

Matsui Y, Hirasawa Y, Sugiura T, Toyoshi T, Kyuki K, and Ito M (2010) Metformin reduces body weight gain and improves glucose intolerance in high-fat diet-fed C57BL/6J mice. Biol Pharm Bull 33:963-970.
Nauck MA, Duran S, Kim D, Johns D, Northrup J, Festa A, Brodows R, and Trautmann M (2007) A comparison of twice-daily exenatide and biphasic insulin aspart in patients with type 2 diabetes who were suboptimally controlled with sulfonylurea and metformin: a non-inferiority study. Diabetologia 50: 259-267.

Nielsen LL, Young AA, and Parkes DG (2004) Pharmacology of exenatide (synthetic exendin-4): a potential therapeutic for improved glycemic control of type 2 diabetes. Regul Pept 117:77-88.

Young AA, Gedulin BR, Bhavsar S, Bodkin N, Jodka C, Hansen B, and Denaro M (1999) Glucose-lowering and insulin-sensitizing actions of exendin-4: studies in obese diabetic $(\mathrm{ob} / \mathrm{ob}, \mathrm{db} / \mathrm{db})$ mice, diabetic fatty Zucker rats, and diabetic rhesus monkeys (Macaca mulatta). Diabetes 48:1026-1034.

Zinman B, Hoogwerf BJ, Durán García S, Milton DR, Giaconia JM, Kim DD, Trautmann ME, and Brodows RG (2007) The effect of adding exenatide to a thiazolidinedione in suboptimally controlled type 2 diabetes: a randomized trial. Ann Intern Med 146:477-485.

Address correspondence to: Shinji Iwasaki, Drug Metabolism and Pharmacokinetics Research Laboratories, Takeda Pharmaceutical Co., Ltd. 35 Landsdowne Street, Cambridge, MA 02139. E-mail: shinji.iwasaki2@ takeda.com 